\title{
An analysis of genetic influence on salivary parameters and caries experience-by correlation of incidence of dental caries and salivary parameters
}

\author{
Abhay Taranath, Pai Deepika*, Sham S Bhat, Sharan Sargod \\ Head\& Professor,Department of Oral \& Macillofacial surgery,Manipal College of Dental Sciences,Manipal \\ Associate professor,Department of Pedodontics \&preventive dentistry, Manipal College of dental sciences, \\ Manipal university, Manipal \\ H.O.D \& Professor, Department of Pedodontics, Yenepoya Dental College, Rajiv Gandhi University of Health Sciences, Karnataka \\ Professor, Department of Pedodontics, Yenepoya Dental College,Rajiv Gandhi University of Health Sciences, Karnataka \\ *Corresponding author E-mail: deepikapai0479@gmail.com
}

\begin{abstract}
Dental Caries is a multifactorial disease where in the interplay of protective and destructive factors causes caries. The twin population is an excellent sample to study the genetic influence on the host factors responsible for caries .The caries experience in twin children is considered to be similar because of similar genetic and environmental predisposition. This study was conducted to analyze the influence of genetic control on caries experience in ten pair of twin children by analyzing the salivary parameters like flow rate, $\mathrm{pH}$, viscosity and buffering capacity and by correlating it to their DMFT, def scores. The results showed no significant statistical difference in the caries experience and the salivary parameters among the twin children that were evaluated .Hence the similar caries experience in twin children can be attributed to their similar genetic and environmental factors.
\end{abstract}

Keywords: Caries experience, salivary parameters, genetic influence.

\section{Introduction}

Genetics plays an important role on causative factors of dental caries especially on the host factors like the tooth structure, immunologic response to the microbes and the salivary factors. The classical approaches to study genetic influence on any particular disease cannot be applied in studying the influence of genetic factors in dental caries, simply because of the uncertainty of assessing equivalent conditions between ancestors, offspring and between siblings.

Therefore the two most possible approaches to link genetic influences in dental caries are the twin study and genetic marker study [1].

The caries experience is considered to be similar in twin children due to the similar genetic and environmental predisposing factors. Similar host factors like the tooth structure, immunologic response to the microbes and the salivary factors contribute to the similarity in the caries experience of twin children. [2] Similar environmental factors like dietary habits and oral hygiene practices do exist among twin children especially when they are reared together [3]. In the past studies were conducted to isolate the influence of genetic factors in causation of dental caries by evaluating the caries experience in twin children who were reared apart. These milestone studies examined the caries experience in twin children that were reared apart by recording their dietary habits, oral hygiene practices and caries indices [4] thus they isolated the influence of environmental factors on genetic factors in development of caries. But today we consider dental caries as a multifactorial disease hence it becomes interesting to analyses the interaction between genetic and environmental factors in causation of dental caries. The study on twin population is also a classical example for the complete complex interaction between the genetic, biologic, environmental, social, behavioral and psychological model of development of caries [5], [6].

Our study was designed to evaluate and correlate caries experience and salivary parameters like flow rate, $\mathrm{pH}$, viscosity and buffering capacity of saliva in twin children reared together. So as to evaluate the genetic influence on salivary factors responsible for caries experience. 


\begin{abstract}
Aim of the study:
1) To assess the caries experience in twin children by recording the DMF/dmf scores.

2) To evaluate the salivary parameters like flow rate, $\mathrm{pH}$, viscosity and buffering capacity in twin children.

3) To correlate the caries experience and salivary parameters in order to relate the genetic control on salivary factors responsible for caries experience.
\end{abstract}

\title{
2 Material and methods
}

After obtaining parent consent and ethical clearance from the concerned authorities 10 identical twin pairs above 6years of age from the primary schools of Mangalore city participated in the study.A detailed oral examination was conducted with the help of a mouth mirror and explorer by two trained dentists. One of the twin child was examined by one dentist while another dentist examined the other child of the twin pair in order to avoid any bias. Their DMF, dmf scores were recorded by two trained dentists separately. A detailed 7 day diet history was obtained by providing a diet diary to each of the twins which was later obtained from the schools and results were tabulated Thier oral hygiene practices were also recorded during their interview at school.

The salivary parameters were evaluated using saliva check kit(GC buffer kit,GC Corpo,India .The saliva evaluation kit contains 20 plastic ,disposable cup for the collection of saliva which is graduated in milliliters , paraffin wax blocks , pipette to withdraw saliva for various tests to be performed, $\mathrm{pH}$ strip, buffer strip which is sealed in aluminium pack to avoid any contact with moisture as it can give false results.Colour coded charts are provided to match the colour change to assess and categorize the $\mathrm{pH}$ and buffering capacity of saliva as per manufacturer's instructions.

The salivary parameters like flow rate, the quantity, $\mathrm{pH}$, buffering capacity and viscosity of stimulated and unstimulated saliva were evaluated.

To estimate the flow rate the lower lip was everted and dried with the use of an absorbent blotting paper, the time taken to wet a new strip of blotting paper that was gently pressed against the lower lip was noted and categorized as in table 1a.

The children were instructed to expectorate any accumulated saliva into the graduated cup for one minute such that it simply drools. The quantity of unstimulated saliva in $\mathrm{ml} /$ second was thus assessed and categorized as in table $1 \mathrm{~b}$.

The children were instructed to chew on a paraffin wax for five minutes and expectorate the stimulated saliva intermittently such that it simply drools into the graduated cup and categorized as in table 1c.

The $\mathrm{pH}$ was determined by withdrawing the saliva with the help of a pipette .One drop of saliva was dispensed on to the $\mathrm{pH}$ strip after about 10 seconds the colour change was matched with the chart provided in the kit and $\mathrm{pH}$ was thus categorized likewise as seen in the table $1 \mathrm{~d}$.

The viscosity of the saliva was categorized as in table 1e.

The buffering capacity of the saliva was estimated by dispensing one drop of saliva each on to the three test pads on the buffer strip provided in the kit. Excess saliva from the test strips were blotted onto an absorbent tissue paper .Colour change on each test pad was observed after about two minutes and the buffer capacity of saliva was categorized as in table $1 f$.

\section{Results}

The data regarding caries experience and various tested salivary parameters obtained were analyzed using chi-square test and Mann-Whitney test .The correlation with their DMFT/dmf scores and salivary parameters were made .The analysis showed that the DMFT scores of the twins , $\mathrm{p}=0.562$ and def scores as $\mathrm{p}=0.569$ as shown in table $2 \mathrm{a}$, were not statistically different. The interpretation of the result showed that caries experience amongst the twin in our study was similar.

The quantity of unstimulated saliva showed $p=0.562$ and stimulated saliva of $p=0.569$ as shown in table $2 b$.The $p H$ of unstimulated and stimulated saliva was $p=0.64$ and $p=0.677$ as shown in table $2 c$. The rate flow of saliva showed $p$ value $=0.98$ as shown in table $2 \mathrm{~d}$. The buffering capacity of saliva showed statistical value of $\mathrm{p}=0.668$ as shown in table 2e. The viscosity of saliva showed statistical value of $\mathrm{p}=0.319$ as shown in table $2 \mathrm{f}$. The salivary parameters showed no significant difference statistically amongst the twin children. The statistical analysis showed no significant difference in the caries experience in twins' children and no statistical difference in the salivary parameters amongst the twins. 


\section{Discussion}

Caries process is a multifactorial complex disease. The outcome of interaction between the risk factors leads to imbalance in oral ecology the end result of which is cavitation and sequalae. [2], [5] Caries risk assessment in an individual remains skeptical because of the role played by genetic factors towards the predisposition of caries is uncertain and needs specific markers to precisely assess the risk of development of caries.

But it is generally agreed upon that susceptibility and resistance to dental caries is inherited. The most convincing data on role of genetics in the pathogenesis of dental caries has been developed by analyzing the caries experience on twin populations. The studies to evaluate the role of genetics in dental caries involved the earliest twin studies which concluded that genetics plays a role in inheritance of dental feature but only as much as it could control the shape of tooth, pit and fissure morphology and position of tooth [3], [4].

Bachrach and Young conducted a study on dental caries in monozygous ("identical") and dizygous ("fraternal") twins in London schools and, although the similarity in caries between twins was found to be greater in monozygous than in dizygous pairs, the difference between the two types of twins was not regarded as statistically significant. These findings were interpreted to suggest that heredity played a minor role in the etiology of dental caries [8].

Goldberg, from a study of 42 pairs of monozygous twins, concluded that heredity influences dental caries indirectly by influencing tooth morphology, especially the formation of pit and fissure systems [3].

Dahlberg and Dahlberg investigated dental caries experienced in twins and concluded that inherited factors are at the least half as important and, at the most, of equal importance as environmental factors in the incidence of this disease.

The differing conclusions reached by Bachrach and Young and Dahlberg and Dahlberg provided the main reason for this study. Furthermore, the environment of twins is inevitably very similar, especially in childhood, and since environmental factors (particularly diet) are known to exert an important influence on the incidence of dental caries, it was thought that a comparison

of the differences in caries experience which occur between unrelated pairs of children and the differences which occur between twins would provide a better estimate of the contribution of environment and hence permit a better evaluation of any possible genetic effects [3], [8].

The advanced studies on genetic influence and predisposition concluded that environmental factors have greater influence but the genetic factors also contribute to the causation of dental caries [9].

Bordoni concluded that there is a strong genetic contribution for caries incidence in primary teeth [9].

Fiarpo conducted a similar study on twin and concluded that the genetic influence on the caries susceptibility was similar in both primary and permanent teeth [10].

Boraas suggested the various inherited traits in caries susceptibility, like the eruption time and sequence of tooth eruption, salivary factors and oral flora, arch shape, dental spacing, propensity of diet [11].

The factors under the genetic influence for caries experience can thus be summarized as:

1) Dental hard tissue which includes enamel biomineralisation, tooth morphology, position and sequence of eruption of the tooth.

2) Immune response, which can reduce the clearance of bacteria from the oral cavity.

3) Inherited deficiency in sugar metabolism that could alter the availability of the substrate.

4) Salivary parameters like the rate of flow, $\mathrm{pH}$ of saliva, buffering capacity of saliva, viscosity of saliva etc.

Our study was designed to establish a relation between the salivary parameters and caries experience in twin children .The statistical analysis suggested that there was no statistical difference in salivary parameters and caries experience in the twin children. The similarity in their environmental factors as well as perhaps the similar genetic predisposition on salivary factors has led to the similarity in the dental caries experience in these twin children.

The rationale explanation for the similarity in caries experience among twin children or even in siblings cannot exclude the role played by the similar dietary habits, oral hygiene practices etc, unless they are reared apart [10], [11] This study is in agreement with the earlier studies that is the caries experience in twin children is not statistically different, however more study is required to clarify the genetic and phenotypic heterogenicity. We thereafter conclude that the caries experience is well correlated to salivary factors which are definitely under the genetic control. Hence salivary factors can be taken into account in analyzing the inherent/genetic risk association to development of dental caries.Saliva is one of the inherited traits that determine the risk of susceptibility or resistance to development of caries.

Table 1a: for assesment of salivary flow rate

\begin{tabular}{ccc} 
& Table 1a: for assesment of salivary flow rate & \\
\hline time in seconds & salivary flow rate \\
\hline less than 30 & high \\
between $30-60$ & normal \\
more than 60 & low \\
\hline
\end{tabular}


Table1b: quantity of unstimulated saliva recorded in 1 minute

quantity of unstimulated saliva recorded in $\mathrm{ml} / \mathrm{min}$

$\begin{array}{cc}<0.3 \mathrm{ml} / \mathrm{min} & \text { low } \\ 0.3-0.7 \mathrm{ml} / \mathrm{min} & \text { normal } \\ >0.7 \mathrm{ml} / \mathrm{min} & \text { high }\end{array}$

Table 1c: quantity of stimulated saliva in $\mathrm{ml}$ collected over 5 minutes quantity of unstimulated saliva recorded in $\mathrm{ml} / \mathrm{min}$

$<3.5 \mathrm{ml}$

between $3.5-5 \mathrm{ml}$

$>5 \mathrm{ml}$ very low

low

normal

Table 1d: for assessment of ph of saliva

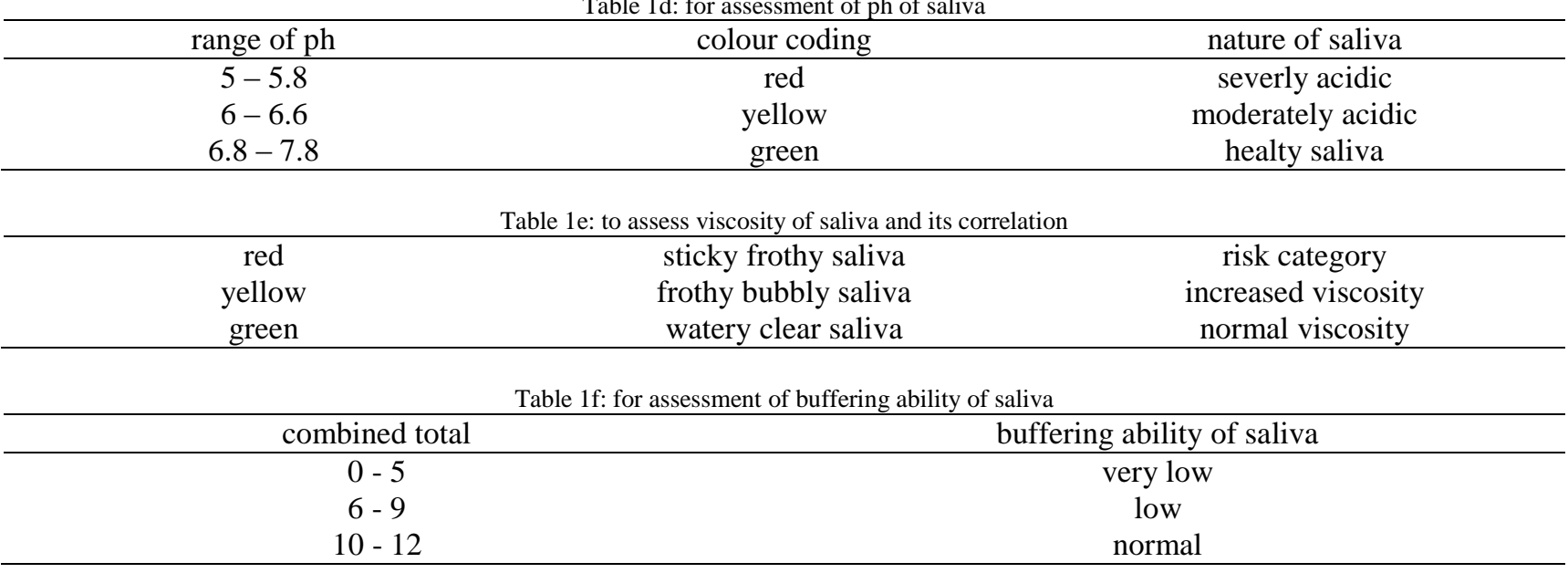

Table 2a: comparison of DMFT and dmf scores between the twin children

\begin{tabular}{|ll|r|r|r|c|}
\hline & TWINS & $\mathrm{N}$ & Mean & Std. Deviation & \multicolumn{1}{c|}{$\mathrm{Z}^{\mathrm{a}}$} \\
\hline DMF & 1.00 & 18 & .444 & .784 & .5010 \\
& 2.00 & 18 & 1.000 & 1.680 & $\mathrm{p}=.616 \mathrm{~ns}$ \\
\hline def. & 1.00 & 18 & 3.111 & 3.160 & .2090 \\
& 2.00 & 18 & 2.833 & 2.834 & $\mathrm{p}=.834 \mathrm{~ns}$ \\
\hline
\end{tabular}

A $\mathrm{Z}=$ Mann- Whitney U test

Table 2b: comparison of Quantity of unstipulated and stimulated saliva in twin children

\begin{tabular}{|ll|r|r|r|r|}
\hline & TWINS & $\mathrm{N}$ & Mean & Std. Deviation & \multicolumn{2}{c|}{$\mathrm{Z}$} \\
\hline US & 1.00 & 10 & .9500 & .2415 & .5800 \\
& 2.00 & 10 & 1.0000 & .1826 & $\mathrm{p}=.562 \mathrm{~ns}$ \\
\hline SS & 1.00 & 10 & 3.9800 & 1.2345 & .5700 \\
& 2.00 & 10 & 3.6400 & 1.5299 & $\mathrm{p}=.569 \mathrm{~ns}$ \\
\hline
\end{tabular}

Table 2c: comparison of $\mathrm{Ph}$ of stimulated and unstimulated saliva in twin children

\begin{tabular}{|ll|r|r|r|rc|}
\hline & TWINS & $\mathrm{N}$ & Mean & Std. Deviation & \multicolumn{2}{|c|}{$\mathrm{Z}$} \\
\hline US-PH & 1.00 & 10 & 6.9000 & .3801 & \multicolumn{2}{|c|}{. } \\
& 2.00 & 10 & 6.8200 & .3584 & $\mathrm{p}=.64 \mathrm{~ns}$ & \\
\hline S-PH & 1.00 & 10 & 7.3400 & .2319 & & .4160 \\
& 2.00 & 10 & 7.2800 & .2700 & $\mathrm{p}=.677 \mathrm{~ns}$ & \\
\hline
\end{tabular}


Table $2 \mathrm{~d}$ comparison of Rateof flow of saliva amoung Twin children

\begin{tabular}{|cl|r|r|r|}
\hline \multirow{2}{*}{} & & \multicolumn{2}{|c|}{ TWINS } & \multirow{2}{*}{ Total } \\
\cline { 3 - 5 } & Count & 1.00 & 2.00 & 3 \\
& $\%$ & 2 & 1 & $15.0 \%$ \\
\hline 30 & Count & $20.0 \%$ & $10.0 \%$ & 15.0 \\
& $\%$ & 1 & 1 & 2 \\
& $\%$ & 5 & $10.0 \%$ & $10.0 \%$ \\
\hline $30--60$ & Count & $50.0 \%$ & $60.0 \%$ & 11 \\
& $\%$ & 1 & 1 & $25.0 \%$ \\
\hline 60 & Count & $10.0 \%$ & $10.0 \%$ & $10.0 \%$ \\
\hline & $\%$ & 1 & 1 & 2 \\
\hline$>60$ & Count & $10.0 \%$ & $10.0 \%$ & $10.0 \%$ \\
\hline & $\%$ & 10 & 10 & 20 \\
\hline Total & Count & $100.0 \%$ & $100.0 \%$ & $100.0 \%$ \\
\hline
\end{tabular}

A. $\mathrm{X} 2=.424 \mathrm{p}=.98 \mathrm{~ns}$

Table 2e comparison of buffering capacity of saliva amoung twin children

\begin{tabular}{|l|r|r|r|r|}
\hline TWINS & $\mathrm{N}$ & Mean & Std. Deviation & \multicolumn{2}{|c|}{$\mathrm{Z}$} \\
\hline 1.00 & 10 & 9.6000 & 2.2706 & \multicolumn{2}{|c|}{.4300} \\
2.00 & 10 & 9.1000 & 2.4244 & $\mathrm{p}=.668 \mathrm{~ns}$ \\
\hline
\end{tabular}

Table $2 \mathrm{f}$ comparison of Viscosity of saliva among twin children

\begin{tabular}{|cl|r|r|r|}
\hline \multirow{2}{*}{} & & \multicolumn{2}{|c|}{ TWINS } & \multirow{2}{*}{ Total } \\
\cline { 2 - 5 } & \multicolumn{1}{|c|}{1.00} & 2.00 & \multicolumn{1}{c|}{14} \\
Frothy bubby & Count & 8 & 6 & $70.0 \%$ \\
\hline Water clear & Count & $80.0 \%$ & $60.0 \%$ & 2 \\
& $\%$ & & 2 & $10.0 \%$ \\
\hline Sticky frothy & Count & & $20.0 \%$ & 4 \\
& $\%$ & 2 & 2 & $20.0 \%$ \\
\hline Total & Count & $20.0 \%$ & $20.0 \%$ & 20 \\
& $\%$ & 10 & 10 & $100.0 \%$ \\
\hline
\end{tabular}

A. $\mathrm{X} 2=2.286 \mathrm{p}=.319 \mathrm{~ns}$

\section{References}

[1] David bixler .Clinical genetics for the dental practitioner.In:Ralph E.McDonald,David R.Avery.Dentistry for the child and adolescent.6th edition.pp82-110.

[2] O.Fejerskov .Changing paradigms in concepts on dental caries: consequences for oral health care .Caries Res 2004; 38:182-191.

[3] MansbridgeJN. Heredity and dental caries. J Dent Res 1959; 38:337-47.

[4] FinnSB, Caldwell RC.Dental caries in twins.A comparison of caries experience of monozygotic twins, dizygotic twins and unrelated children .Arch Oral Biol 1963;8:571-85

[5] Domenick Thomas Zero. Cariology - Dental caries process: Dental Clinics of North America 1999; 43:635-638.

[6] Conry JP, Messer LB, Boraas JC, Aeppli DP, Bouchard TJ. Dental caries and treatment characteristics in human twins reared apart. Arch Oral Biol 1993; 38:937-43.

[7] Niswander JD. Effects of heredity and environment on development of dentition. J Dent Res1963; 42:1288-98.

[8] Bachrach, F. H., and Young, M. A.: Comparison of the Degree of Resemblance in Dental Characters Shown in Pairs of Twins of Identical and Fraternal Types, B D. J1927; 48: 1293- 1304.

[9] BordoniN, Dono R,Manfredi C,Allegrotti I . Prevelance of dental caries in twin's .J Dent Children 1973; 40:440-3. 
[10] FairpoCG.Total caries experience in monozygotic and like sexed dizygotic twins of caucasoid origin aged 5-15 years. Arch Oral Biol 1979; 24:491-4.

[11] BoraasJC, Messer LB,Till MJ. A genetic contribution to dental caries, occlusion and morphology as demonstrated by twins rared apart J Dent Res 1988; 67:1150-5. 